\title{
Outage probability and ergodic capacity analysis in power splitting based full-duplex relaying networks of double rayleigh fading channel for vehicular communications
}

\author{
Phu Tran Tin ${ }^{1}$, Duy-Hung $\mathrm{Ha}^{2}$, Q. S. Vu ${ }^{3}$, Tran Thanh Trang ${ }^{4}$ \\ ${ }^{1}$ Faculty of Electronics Technology, Industrial University of Ho Chi Minh City, Vietnam \\ ${ }^{2}$ Wireless Communications Research Group, Faculty of Electrical and Electronics Engineering, \\ Ton Duc Thang University, Vietnam \\ ${ }^{3}$ Faculty of Electronics and Automation, Hongbang International University, Vietnam \\ ${ }^{4}$ National Key Laboratory of Digital Control and System Engineering, Vietnam
}

\section{Article Info}

Article history:

Received Jul 3, 2019

Revised Sep 9, 2019

Accepted Oct 2, 2019

\section{Keywords:}

Double rayleigh fading channel

Energy harvesting $(\mathrm{EH})$

Full-duplex (FD)

Monte carlo simulation

Relaying network

\begin{abstract}
In this research, we present and investigate the system performance analysis in terms of the outage probability (OP) and ergodic capacity (EC) of the Fullduplex (FD) relaying networks via double Rayleigh fading channel. In this system model, we propose the power splitting (PS) protocol for the vehicular communications network. Firstly, we analyze and derive the integral form of the system OP and EC. Then, the correctness of the OP and EC expressions are verified by the Monte Carlo simulation. Finally, we can see that the analytical and the simulation results are matched with each other in the connection with the main system parameter.
\end{abstract}

Copyright $\left({ }_{0} 2020\right.$ Institute of Advanced Engineering and Science. All rights reserved.

\section{Corresponding Author:}

Duy-Hung Ha,

Wireless Communications Research Group,

Faculty of Electrical and Electronics Engineering,

Ton Duc Thang University,

Ho Chi Minh City, Vietnam.

Email: haduyhung@tdtu.edu.vn

\section{INTRODUCTION}

In recent years, wireless transmission has experienced rapid development. Relaying has been proved to be an efficient way to extend the coverage area of wireless networks and increase transmission reliability without additional transmit power at the transmitters. However, the forwarding operations at the relay still require extra energy. For relay nodes powered by limited power batteries instead of the regular power grid, such as mobile devices, such extra energy consumption may cause serious concerns. Recently, harvesting energy from ambient radio-frequency (RF) energy was proposed, and it has been well studied. The idea that receiving information and harvesting energy simultaneously is not only appealing but also worth further investigating. The recent works suggested that data transmitting and battery charging could be fulfilled at the same time, and therefore, the combining of energy harvesting module and relay could be an available solution of extra energy consumption problem at the relay [1-6]. Recently, deploying energy harvesting (EH) relays that utilize the energy harvested from the source signal for data transfer has received considerable attention. With EH capabilities, relays can be installed conveniently without wiring cost and the need for battery replacement. Besides, substantial transmission power can be saved because the inter-node distance is shortened using multihop transmissions. However, information relaying consumes additional resources (i.e., time and bandwidth) compared to the direct transmission and hence some early work has been conducted 
focusing on assessing the feasibility of EH relays. The gain offered by the EH relay based on time switching relaying (TSR) is analyzed theoretically in [7] for both decode-and-forward (DF) and amplify-and-forward (AF) relaying modes with a significant gain. The EH relay based on DF is also considered in [8], where the relay determines whether to perform $\mathrm{EH}$ or information relaying before the source transmission according to a greedy policy. Moreover, an analytical framework is proposed in [9] to evaluate the performance of the $\mathrm{EH}$ relay based on AF for both TS and power splitting (PS) protocols under the effect of the critical system parameters such as noise variances, source to relay distance, transmission rate, and energy conversion efficiency. Moreover, relay selection (RS) is a practical approach to balance the tradeoff between reliability improvement and spectral efficiency loss due to information repetition [10]. In [11], two RS schemes aiming to attain the optimal tradeoff between energy transfer and outage probability/ergodic capacity for DF relays are studied.

In this paper, the outage probability (OP) and ergodic capacity (EC) analysis of the Full-duplex (FD) relaying networks via double Rayleigh fading channel is proposed and investigated. We investigate the system model in the power splitting protocol for the vehicular communications network. The integral form of OP and EC is derived in connection with all primary parameters. Finally, the analytical expressions are verified by Monte Carlo simulation. From the research results, we can state that the analytical and the simulation results are matched with each other. This paper can provide a recommendation for relaying communication networks. Here are the main contributions of this research:

a) The Full-duplex (FD) relaying networks via double Rayleigh fading channel is proposed and investigated

b) The integral form of OP and EC is derived.

c) The correctness of the system performance is verified by the Monte Carlo simulation in the connection with the main system parameters.

The structure of this paper is proposed as follows. Sections 2 shows the system model of the relaying network. Sections 3 derives the analytical expressions of OP and EC of the model system. Section 4 gives numerical results and some discussions. Finally, Section 5 concludes this research.

\section{SYSTEM MODEL}

As shown in Figure 1, the FD relaying network via double Rayleigh fading channel. In Figure 1, the source (S) and destination (D) communicate via helping of the energy-constrained intermediate relay (R). The PS protocol of the system model is drawn in Figure 2. In Figure 2, we denote $\mathrm{T}$ is the block time in which the $\mathrm{S}$ fully transmit the information data to the $\mathrm{D}$. In this protocol, the $\mathrm{R}$ harvests $\rho \mathrm{P}_{\mathrm{s}}$ energy from the $S$ and use $(1-\rho) P_{s}$ energy to transfer information from the $S$ to the $D$. Here, we denote $\rho$ is the power splitting factor, which $\rho \in(0,1)$ [12-20].

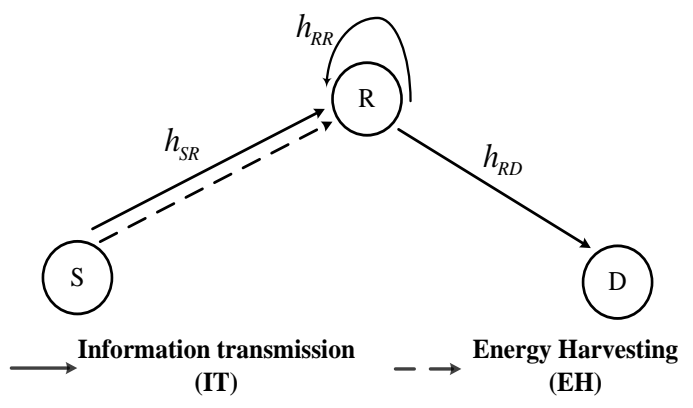

Figure 1. System model

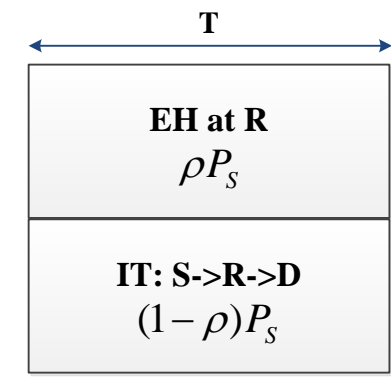

Figure 2. The power splitting (PS) protocol

\subsection{Energy Harvesting Phase}

In $\mathrm{EH}$ phase, the received signal at the $\mathrm{R}$ node is

$$
y_{r}=\sqrt{\rho P_{s}} h_{S R} x_{s}+n_{r}
$$


Where $x_{s}$ is the energy symbol and must be satisfied $\mathrm{E}\left\{\left|x_{s}\right|^{2}\right\}=1$ which $\mathrm{E}\{\bullet\}$ is the expectation operator. $\mathrm{P}_{\mathrm{S}}$ is the transmit power of source and $0<\rho<1$ is power splitting factor. $\mathrm{n}_{\mathrm{r}}$ is the additive white Gaussian noise (AWGN) at the relay node with variance $\mathrm{N}_{0} . h_{S R}$ is double Rayleigh channel.

From (1), the average transmitted power at the $\mathrm{R}$ can be calculated by

$$
P_{r}=\frac{E_{r}}{T}=\eta \rho P_{s}\left|h_{S R}\right|^{2}
$$

where $0<\eta \leq 1$ is energy conversion efficiency.

\subsection{Information transmission phase}

In the IT phase, the received signal at the $\mathrm{R}$ can be given as

$$
y_{r}=\sqrt{(1-\rho) P_{s}} h_{S R} x_{s}+h_{R R} x_{r}+n_{r}
$$

where $x_{r}$ is transmitted signal and also must be satisfied $\mathrm{E}\left\{\left|x_{r}\right|^{2}\right\}=1$ and $h_{R R}$ is loopback interference channel and modeled by double Rayleigh channel.

\section{THE SYSTEM PERFORMANCE}

In our model, we mostly focus on decode and forward (DF) protocol. Hence, the signal to interference noise ratio (SINR) at the relay can be obtained as from (3),

$$
\gamma_{1}=\frac{(1-\rho) P_{s}\left|h_{S R}\right|^{2}}{P_{r}\left|h_{R R}\right|^{2}+N_{0}}
$$

substituting (2) into (4), $\gamma_{1}$ can be reformulated as

$$
\gamma_{1} \approx \frac{1-\rho}{\eta \rho\left|h_{R R}\right|^{2}}
$$

In this time slot also, the received signal at the destination can be expressed as

$$
y_{d}=\sqrt{P_{r}} h_{R D} x_{r}+n_{d}
$$

where $\mathrm{n}_{\mathrm{d}}$ is the AWGN at destination node with variance $\mathrm{N}_{0} . h_{R D}$ is channel gain between R-D link and modeled by double Rayleigh channel.

From (6), the SINR at the destination can be claimed as

$$
\gamma_{2}=\frac{P_{r}\left|h_{R D}\right|^{2}}{N_{0}}
$$

substituting (2) into (7), $\gamma_{2}$ can be rewritten as

$$
\gamma_{2}=\eta \rho \Delta\left|h_{S R}\right|^{2}\left|h_{R D}\right|^{2}
$$

\subsection{Ergodic Capacity (EC) analysis}

In this section, we need to evaluate the ergodic capacity from the source to relay $C_{r}$ and for relay to destination link $C_{d}$ in order to can obtain the capacity of the system $C_{D F}=\min \left(C_{r}, C_{d}\right)$. After that $C_{r}$ and $C_{d}$ are given by the following equations as in [13]. 


$$
\begin{aligned}
& C_{r}=\frac{1}{\ln 2} \int_{0}^{\infty} \frac{1-F_{\gamma_{1}}(x)}{1+x} d x \\
& C_{d}=\frac{1}{\ln 2} \int_{0}^{\infty} \frac{1-F_{\gamma_{2}}(x)}{1+x} d x
\end{aligned}
$$

At first, we have to find the cumulative density function (CDF) for (9) and (10), respectively. Followed by definition of CDF, we have $F_{\gamma_{1}}(x)=\operatorname{Pr}\left(\gamma_{1}<x\right)$ and $F_{\gamma_{2}}(x)=\operatorname{Pr}\left(\gamma_{2}<x\right)$.

Remark 1. The probability density function (PDF) and cumulative distribution function (CDF) of double Rayleigh channel. As shown in [20], the CDF and PDF of the random variable (RV) $\left|h_{S R}\right|^{2},\left|h_{R D}\right|^{2}$ and $\left|h_{R R}\right|^{2}$ can be obtained as, respectively.

$$
F_{\left|h_{i}\right|^{2}}(x)=1-2 \sqrt{x} K_{1}(2 \sqrt{x}), f_{\left|h_{i}\right|^{2}}(x)=2 K_{0}(2 \sqrt{x})
$$

where $K_{v}(\bullet)$ is the modified Bessel function of the second kind and $\mathrm{v}^{\text {th }}$ order and $i \in(S R, R D, R R)$

From (9), we will find $F_{\gamma_{1}}(x)$ as followings:

$$
F_{\gamma_{1}}(x)=\operatorname{Pr}\left(\gamma_{1}<x\right)
$$

substituting (5) into (12), we have:

$$
F_{\gamma_{1}}(x)=\operatorname{Pr}\left(\frac{1-\rho}{\eta \rho\left|h_{R R}\right|^{2}}<x\right)=\operatorname{Pr}\left(\left|h_{R R}\right|^{2}>\frac{1-\rho}{\eta \rho x}\right)=1-\operatorname{Pr}\left(\left|h_{R R}\right|^{2} \leq \frac{1-\rho}{\eta \rho x}\right)=1-F_{\left|h_{R R}\right|^{2}}\left(\frac{1-\rho}{\eta \rho x}\right)
$$

combine (11), (13) can be reformulated as:

$$
F_{\gamma_{1}}(x)=2 \sqrt{\frac{1-\rho}{\eta \rho x}} K_{1}\left(2 \sqrt{\frac{1-\rho}{\eta \rho x}}\right)
$$

substituting (14) into (9), the EC between S-Rlink can be obtained as:

$$
C_{r}=\frac{1}{\ln 2} \int_{0}^{\infty} \frac{1-2 \sqrt{\frac{1-\rho}{\eta \rho x}} K_{1}\left(2 \sqrt{\frac{1-\rho}{\eta \rho x}}\right)}{1+x} d x
$$

apply $\frac{1}{1+x}=G_{1,1}^{1,1}\left(\left.x\right|_{0} ^{0}\right)$ in [21] Equation 9.3, where $G_{p, q}^{m, n}(. \mid \ldots)$ is the Meijer function.

In (15) can be rewritten as the following:

$$
C_{r}=\frac{1}{\ln 2} \int_{0}^{\infty} G_{1,1}^{1,1}\left(\left.x\right|_{0} ^{0}\right)\left\{1-2 \sqrt{\frac{1-\rho}{\eta \rho x}} K_{1}\left(2 \sqrt{\frac{1-\rho}{\eta \rho x}}\right)\right\} d x
$$

similar, from (10) we have:

$$
\begin{aligned}
F_{\gamma_{2}}(x) & =\operatorname{Pr}\left(\gamma_{2}<x\right)=\operatorname{Pr}\left(\eta \rho \Delta\left|h_{S R}\right|^{2}\left|h_{R D}\right|^{2}<x\right) \\
& =\operatorname{Pr}\left(\left|h_{S R}\right|^{2}<\frac{x}{\eta \rho \Delta\left|h_{R D}\right|^{2}}\right)=\int_{0}^{\infty} F_{\left|h_{S R}\right|^{2}}\left(\left.\frac{x}{\eta \rho \Delta\left|h_{R D}\right|^{2}}|| h_{R D}\right|^{2}=y\right) f_{\left|h_{R D}\right|^{2}}(y) d y
\end{aligned}
$$

combine (11), (17) can be claimed as: 


$$
F_{\gamma_{2}}(x)=1-4 \int_{0}^{\infty} \sqrt{\frac{x}{\eta \rho \Delta y}} K_{1}\left(2 \sqrt{\frac{x}{\eta \rho \Delta y}}\right) \times K_{0}(2 \sqrt{y}) d y
$$

substituting (18) into (10), we have:

$$
C_{d}=\frac{4}{\ln 2} \int_{0}^{\infty} \int_{0}^{\infty} G_{1,1}^{1,1}\left(\left.x\right|_{0} ^{0}\right) \sqrt{\frac{x}{\eta \rho \Delta y}} K_{1}\left(2 \sqrt{\frac{x}{\eta \rho \Delta y}}\right) \times K_{0}(2 \sqrt{y}) d x d y
$$

finally, we can obtain the end to end EC of the system as the following :

$$
C=\min \left\{\begin{array}{l}
\frac{1}{\ln 2} \int_{0}^{\infty} G_{1,1}^{1,1}\left(\left.x\right|_{0} ^{0}\right)\left\{1-2 \sqrt{\frac{1-\rho}{\eta \rho x}} K_{1}\left(2 \sqrt{\frac{1-\rho}{\eta \rho x}}\right)\right\} d x, \\
\frac{4}{\ln 2} \int_{0}^{\infty} \int_{0}^{\infty} G_{1,1}^{1,1}\left(\left.x\right|_{0} ^{0}\right) \sqrt{\frac{x}{\eta \rho \Delta y}} K_{1}\left(2 \sqrt{\frac{x}{\eta \rho \Delta y}}\right) \times K_{0}(2 \sqrt{y}) d x d y
\end{array}\right\}
$$

\subsection{Outage probability $(\mathrm{OP})$ analysis}

The OP of the system can be defined as

$$
O P=\operatorname{Pr}\left(\gamma_{e 2 e}<\gamma_{t h}\right)=\operatorname{Pr}\left[\min \left(\gamma_{1}, \gamma_{2}\right)<\gamma_{t h}\right]
$$

where $\gamma_{e 2 e}=\min \left(\gamma_{1}, \gamma_{2}\right)$ and $\gamma_{t h}=2^{R}-1$ is the threshold, R: source rate.

Substituting (7), (8) into (21), finally, we have

$$
\begin{aligned}
O P & =\operatorname{Pr}\left[\min \left(\frac{1-\rho}{\eta \rho\left|h_{R R}\right|^{2}}, \eta \rho \Delta\left|h_{S R}\right|^{2}\left|h_{R D}\right|^{2}\right)<\gamma_{t h}\right]= \\
& =1-\operatorname{Pr}\left(\frac{1-\rho}{\eta \rho\left|h_{R R}\right|^{2}} \geq \gamma_{t h}\right) \operatorname{Pr}\left(\eta \rho \Delta\left|h_{S R}\right|^{2}\left|h_{R D}\right|^{2} \geq \gamma_{t h}\right)
\end{aligned}
$$

the first term of (22) can be computed as

$$
\operatorname{Pr}\left(\frac{1-\rho}{\eta \rho\left|h_{R R}\right|^{2}} \geq \gamma_{t h}\right)=\operatorname{Pr}\left(\left|h_{R R}\right|^{2} \leq \frac{1-\rho}{\gamma_{t h} \eta \rho}\right)=1-2 \sqrt{\frac{1-\rho}{\gamma_{t h} \eta \rho}} K_{1}\left(2 \sqrt{\frac{1-\rho}{\gamma_{t h} \eta \rho}}\right)
$$

and the second term of (22)can be calculated as

$$
\operatorname{Pr}\left(\eta \rho \Delta\left|h_{S R}\right|^{2}\left|h_{R D}\right|^{2} \geq \gamma_{t h}\right)=1-\operatorname{Pr}\left(\eta \rho \Delta\left|h_{S R}\right|^{2}\left|h_{R D}\right|^{2}<\gamma_{t h}\right)
$$

apply the result of (18), (24) can be reformulated as

$$
\operatorname{Pr}\left(\eta \rho \Delta\left|h_{S R}\right|^{2}\left|h_{R D}\right|^{2} \geq \gamma_{t h}\right)=4 \int_{0}^{\infty} \sqrt{\frac{\gamma_{t h}}{\eta \rho \Delta y}} K_{1}\left(2 \sqrt{\frac{\gamma_{t h}}{\eta \rho \Delta y}}\right) \times K_{0}(2 \sqrt{y}) d y
$$

substituting (23), (25) into (22), finally, the OP of the system can be claimed as

$$
O P=1-4\left\{1-2 \sqrt{\frac{1-\rho}{\gamma_{t h} \eta \rho}} K_{1}\left(2 \sqrt{\frac{1-\rho}{\gamma_{t h} \eta \rho}}\right)\right\} \times \int_{0}^{\infty} \sqrt{\frac{\gamma_{t h}}{\eta \rho \Delta y}} K_{1}\left(2 \sqrt{\frac{\gamma_{t h}}{\eta \rho \Delta y}}\right) \times K_{0}(2 \sqrt{y}) d y
$$




\section{NUMERICAL RESULTS AND DISCUSSION}

In this section, we use Monte Carlo to verify the analytical expressions as in [22-25]. As shown in Figure 3 and 4 , the OP and EC versus $\Delta$ with the primary parameters as $\rho=0.85,0.5 ; \eta=0.8$ and $R=0.5$. From Figure 3 and 4, the OP of the model system has a significant decrease, but the EC has a considerable increase while we vary the $\Delta$ from $-5 \mathrm{~dB}$ to $25 \mathrm{~dB}$. From the results, we can convince that the analytical and simulation results are the same with all values of $\Delta$.

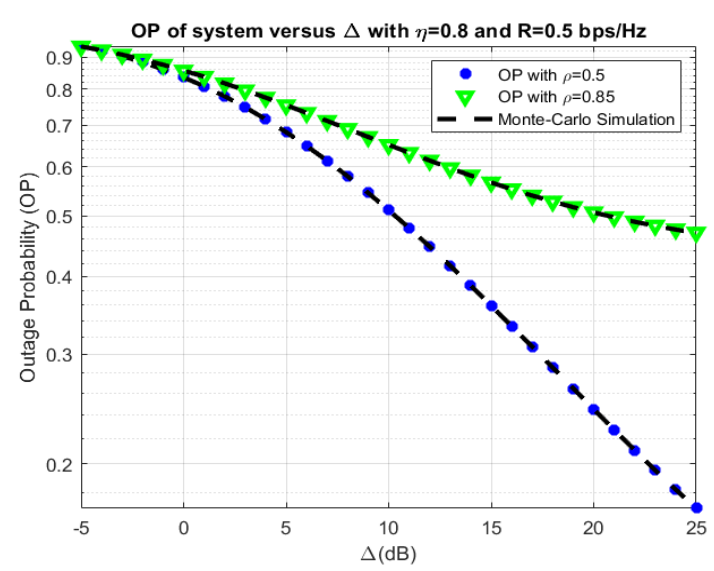

Figure 3. OP versus $\Delta$

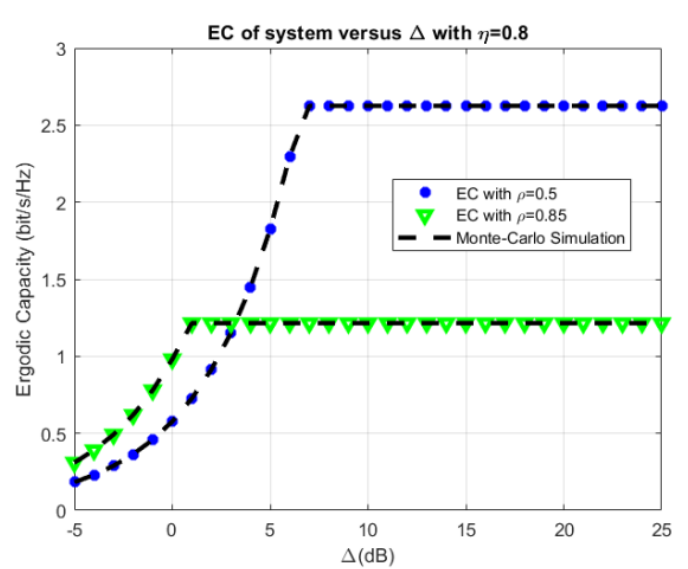

Figure 4. EC versus $\Delta$

Furthermore, the influence of the energy efficiency on the OP and EC is investigated in Figure 5 and 6 . In these cases, we set the primary parameters as the following $\Delta=0.5,0.7 ; \rho=0.5$; and $\mathrm{R}=0.5$. The OP of system network decreases significantly but the EC increases while the energy efficiency varies from 0 to 1. It can be observed that the more energy efficiency transfers the more EC and less OP of the system network. In addition, the simulation curve matches well with the analytical curve.

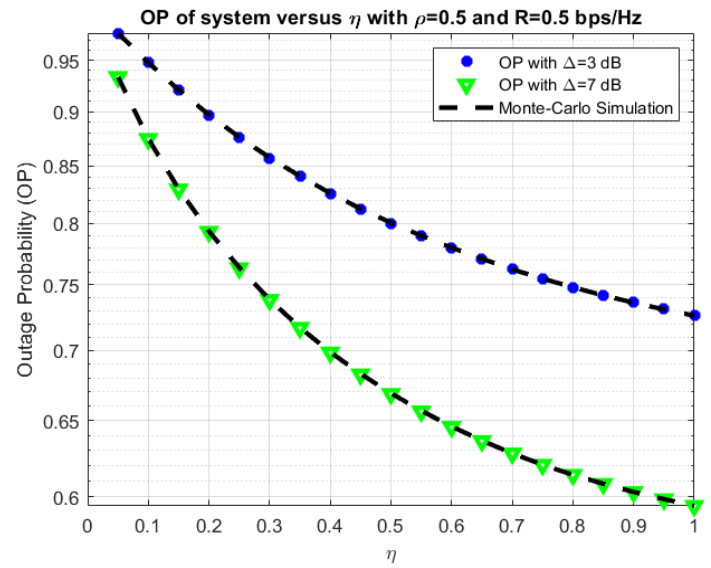

Figure 5. OP versus $\eta$

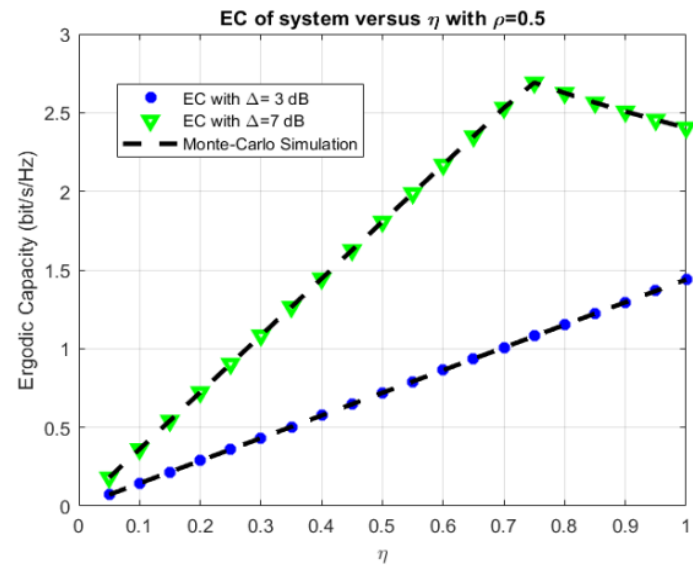

Figure 6. EC versus $\eta$

Finally, the effect of the power splitting factor $\rho$ on the OP and EC of the system network with the main parameters as $\eta=0.5,0.9 ; \Delta=0.5$ and $R=0.5$ is illustrated in Figure 7,8 . From Figure 7 , we can see that the OP od the system network decreases in the first interval values of $\rho$, and after that, the OP has an increase. The optimal value of the OP can be obtained with $\rho$ from 0.6 to 0.7 . In the same way, the EC has a considerable increase when $\rho$ varies from 0 to the optimal value of $\rho$, and then has a massive decrease. The optimal value of EC can be obtained with $\rho$ from 0.5 to 0.7 . Moreover, the analytical values agree well with the simulation values to verify the correctness of the analytical expressions. 


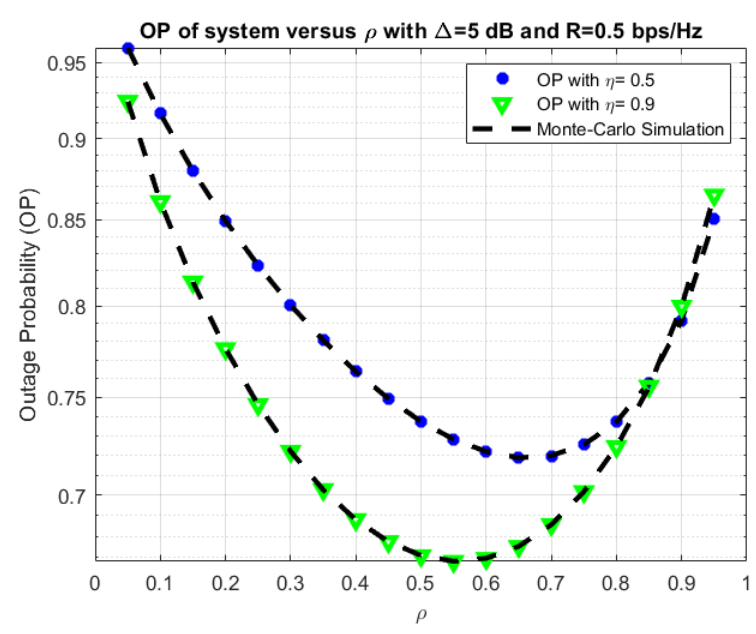

Figure 7. OP versus $\rho$

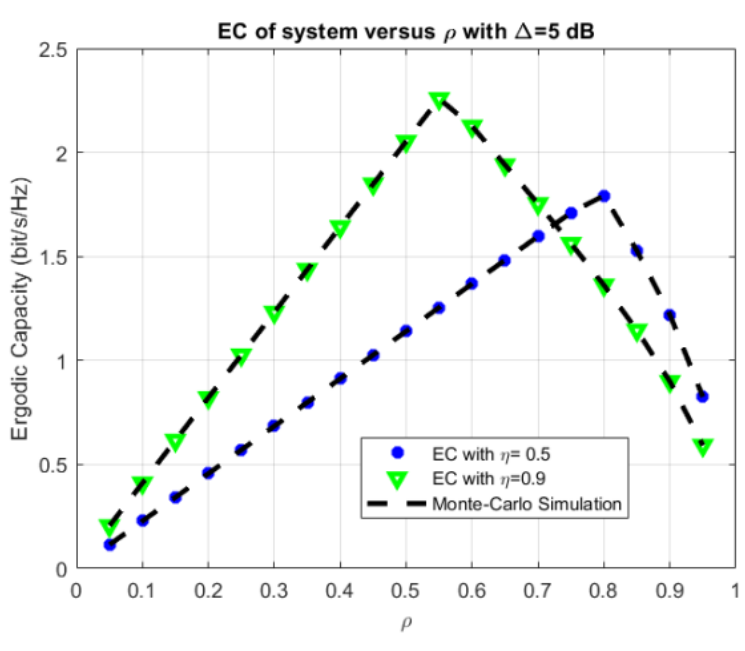

Figure 8. EC versus $\rho$

\section{CONCLUSION}

In this paper, the OP and EC analysis of the FD relaying networks via a double Rayleigh fading channel is proposed and investigated. We investigate the system model in the power splitting protocol for the vehicular communications network. The integral form of OP and EC is derived in connection with all primary parameters. Finally, the analytical expressions are verified by Monte Carlo simulation. From the research results, we can state that the analytical and the simulation results are matched with each other. This paper can provide a recommendation for relaying communication networks.

\section{ACKNOWLEDGMENTS}

This research was supported by National Key Laboratory of Digital Control and System Engineering (DCSELAB), HCMUT, VNU-HCM, Vietnam.

\section{REFERENCES}

[1] Chen, He, Chao Zhai, Yonghui Li, and Branka Vucetic. "Cooperative Strategies for Wireless-Powered Communications: An Overview." IEEE Wireless Communications 25, no. 4 (08 2018): 112-19. doi:10.1109/mwc.2017.1700245.

[2] Bi, S., Ho, C. K., \& Zhang, R. "Wireless powered communication: Opportunities and challenges". IEEE Communications Magazine, 2015, 53, 117-125.

[3] Niyato, D., Kim, D. I., Maso, M., \& Han, Z. "Wireless Powered Communication Networks: Research Directions and Technological Approaches“. IEEE Wireless Communications, 2017,2-11.

[4] Yu, H., Lee, H., \& Jeon, H. (2017, 10). "What is 5G? Emerging 5G Mobile Services and Network Requirements". Sustainability, 2017, 9, 1848.

[5] C. R. Valenta and G. D. Durgin, "Harvesting wireless power: Survey of energy-harvester conversion efficiency in far-field, wireless power transfer systems,'” IEEE Microw. Mag., vol. 15, no. 4, pp. 108-120, Jun. 2014.

[6] Lai, Xiazhi, Wanxin Zou, Xutao Li, and Lisheng Fan. "Multiuser Energy Harvesting Relaying System with Direct Links." IET Communications 11, no. 12 (08, 2017): 1846-852. doi:10.1049/iet-com.2016.1288.

[7] Kawabata, Hiroki, and Koji Ishibashi. "RF Energy Powered Feedback-aided Cooperation." 2014 IEEE 25th Annual International Symposium on Personal, Indoor, and Mobile Radio Communication (PIMRC), 092014. doi:10.1109/pimrc.2014.7136211.

[8] Krikidis, Ioannis, Stelios Timotheou, and Shigenobu Sasaki. "RF Energy Transfer for Cooperative Networks: Data Relaying or Energy Harvesting?" IEEE Communications Letters 16, no. 11 (11 2012): 1772-775. doi:10.1109/lcomm.2012.091712.121395.

[9] Nasir, Ali A., Xiangyun Zhou, Salman Durrani, and Rodney A. Kennedy. "Relaying Protocols for Wireless Energy Harvesting and Information Processing. "IEEE Transactions on Wireless Communications 12, no. 7 (07 2013): 3622-636. doi:10.1109/twc.2013.062413.122042.

[10] Laneman, J.n., D.n.c. Tse, and G.w. Wornell. "Cooperative Diversity in Wireless Networks: Efficient Protocols and Outage Behavior." IEEE Transactions on Information Theory 50, no. 12 (12 2004): 3062-080. doi:10.1109/tit.2004.838089. 
[11] Michalopoulos, Diomidis S., Himal A. Suraweera, and Robert Schober. "Relay Selection for Simultaneous Information Transmission and Wireless Energy Transfer: A Tradeoff Perspective." IEEE Journal on Selected Areas in Communications, 2015, 1. doi:10.1109/jsac.2015.2391771.

[12] Tan N. Nguyen, T.H.Q.Minh, Phuong T. Tran and Miroslav Voznak. "Energy Harvesting over Rician Fading Channel: A Performance Analysis for Half-Duplex Bidirectional Sensor Networks under Hardware Impairments". Sensors, 2018, 18.

[13] Tan N. Nguyen, T.H.Q.Minh, Phuong T. Tran and Miroslav Voznak. "Adaptive Energy Harvesting Relaying Protocol for Two-Way Half Duplex System Network over Rician Fading Channel“. Wireless Communications and Mobile Computing, 2018.

[14] Tin, Phu Tran, Tran Hoang Quang Minh, Tan N. Nguyen, and Miroslav Voznak. "System Performance Analysis of Half-Duplex Relay Network over Rician Fading Channel." TELKOMNIKA (Telecommunication, Computing, Electronics and Control). 16, no. 1 (02, 2018): 189. doi:10.12928/telkomnika.v16il.7491.

[15] Rashid, Tarique, Sunil Kumar, Akshay Verma, Prateek Raj Gautam, and Arvind Kumar. "Pm-EEMRP: Postural Movement Based Energy Efficient Multi-hop Routing Protocol for Intra Wireless Body Sensor Network (IntraWBSN)." TELKOMNIKA Telecommunication, Computing, Electronics and Control), 16, no. 1 (02, 2018): 166. doi:10.12928/telkomnika.v16i1.7318.

[16] A. F. Morabito, "Power Synthesis of Mask-Constrained Shaped Beams Through Maximally-Sparse Planar Arrays," TELKOMNIKA (Telecommunication Computing Electronics and Control), vol. 14, n. 4, pp. 1217-1219, 2016.

[17] Nguyen, T. N., T. T. Duy, G.-T. Luu, P. T. Tran, and M. Voznak. "Energy Harvesting-based Spectrum Access with Incremental Cooperation, Relay Selection and Hardware Noises." Radioengineering 26, no. 1 (04, 2017): 240-50. doi:10.13164/re.2017.0240.

[18] Mckay, Matthew R., Alex J. Grant, and Iain B. Collings. "Performance Analysis of MIMO-MRC inDoubleCorrelated Rayleigh Environments."IEEE Transactions on Communications 55, no. 3 (03 2007): 497-507. doi:10.1109/tcomm.2007.892450.

[19] Tran Hoang Quang Minh. "Hybrid Time-Power Switching Protocol of Energy Harvesting Bidirectional Relaying Network: Throughput and Ergodic Capacity Analysis." TELKOMNIKA Telecommunication, Computing, Electronics and Control), 16, no. 5 (10, 2018): 189. http://dx.doi.org/10.12928/telkomnika.v16i5.9118.

[20] M. Seyfi et al., "Relay selection in dual-hop vehicular networks," IEEE Signal Process. Lett., vol. 18, no. 2, pp. 134-137, Feb. 2011.

[21] Table of Integrals, Series, and Products, 2015

[22] Nguyen, Tan N., Miroslav Voznak, Tran Hoang Quang Minh, Phuong T. Tran, Phu Tran Tin, and Thanh-Long Nguyen. "Outage Probability Analysis of Power Splitting Power-Beacon Assisted Energy Harvesting Relay Wireless Communication Networks." 2018 IEEE/ACM 22nd International Symposium on Distributed Simulation and Real Time Applications (DS-RT), 10 2018. doi:10.1109/distra.2018.8600930.

[23] Tin, Phu Tran, Minh Tran, Tan N. Nguyen, and Thanh-Long Nguyen. "A New Look at Energy Harvesting Halfduplex DF Power Splitting Protocol Relay Network over Rician Channel in Case of Maximizing Capacity." Indonesian Journal of Electrical Engineering and Computer Science (IJEECS) 13, no. 1 (01, 2019): 249. doi:10.11591/ijeecs.v13.i1.pp249-257.

[24] Tin, Phu Tran, Le Anh Vu, Tan N. Nguyen, and Thanh-Long Nguyen. "User Selection Protocol in DF Cooperative Networks with Hybrid TSR-PSR Protocol Based Full-duplex Energy Harvesting over Rayleigh Fading Channel: System Performance Analysis." Indonesian Journal of Electrical Engineering and Computer Science (IJEECS) 13, no. 2 (02, 2019): 534. doi:10.11591/ijeecs.v13.i2.pp534-542.

[25] Tin, Phu Tran, Minh Tran, Tan N. Nguyen, and Thanh-Long Nguyen. "System Performance Analysis of Hybrid Time-power Switching Protocol of EH Bidirectional Relaying Network in Amplify-and-forward Mode." Indonesian Journal of Electrical Engineering and Computer Science (IJEECS), 14, no. 1 (04, 2019): 118. doi:10.11591/ijeecs.v14.i1.pp118-126. 